\title{
Evidence-based ergonomics: a model and conceptual structure proposal
}

\author{
Dierci Marcio Silveira \\ Industrial Systems, Petroleum, Gas and Energy Laboratory - LABSE, Industrial and \\ Metallurgical Engineering School,Universidade Federal Fluminens-UFF, Av. dos Trabalhadores, \\ 420 - Volta Redonda-RJ-Brazil CEP 27255-125 E-mail: dsilveira@metal.eeimvr.uff.br
}

\begin{abstract}
In Human Factors and Ergonomics Science (HFES), it is difficult to identify what is the best approach to tackle the workplace and systems design problems which needs to be solved, and it has been also advocated as transdisciplinary and multidisciplinary the issue of "How to solve the human factors and ergonomics problems that are identified?". The proposition on this study is to combine the theoretical approach for Sustainability Science, the Taxonomy of the Human Factors and Ergonomics (HFE) discipline and the framework for Evidence-Based Medicine in an attempt to be applied in Human Factors and Ergonomics. Applications of ontologies are known in the field of medical research and computer science. By scrutinizing the key requirements for the HFES structuring of knowledge, it was designed a reference model, First, it was identified the important requirements for HFES Concept structuring, as regarded by Meister. Second, it was developed an evidence-based ergonomics framework as a reference model composed of six levels based on these requirements. Third, it was devised a mapping tool using linguistic resources to translate human work, systems environment and the complexities inherent to their hierarchical relationships to support future development at Level 2 of the reference model and for meeting the two major challenges for HFES, namely, identifying what problems should be addressed in HFE as an Autonomous Science itself and proposing solutions by integrating concepts and methods applied in HFES for those problems.
\end{abstract}

Keywords: Ergonomics Modeling, Evidence, Taxonomy, Ontology, Validity

\section{Introduction}

This paper describes a reflexive approach on the importance of consistent evidence application of results from Human Factors and Ergonomics studies and the feasibility to develop an Evidence-Based Ergonomics supportive to the sustainability of socio-technical and ecological systems, its conceptual understanding, its practice, and how it can be transferred to provide knowledge-type information about daily practitioner activities. If an ergonomics and human factors program is under development consideration, what is the evidence addressing ergonomics research and practice that one can get about from an improved approach and general and particular outcomes?

Evidence-Based Ergonomics may be described by the process of systematically reviewing, appraising and applying field studies and research findings to aid the development and the implementation ergonomics solutions in a variety of demands and contexts, such as the development of ergonomics educational programs. 
Several insights from the research into sustainability from an ergonomics perspective are particularly relevant to define and to provide the notion that the truly ergonomic problem cannot be adequately "captured" or represented from any single perspective. Indeed, when we speak of sustainability in socio-technical systems, for instance, the sheer diversity of elements and interactions requires a plurality of perspectives. In this case, we are referring to systemic ways of organizing our thinking about complex social-ecological reality within which we live.

By adopting Evidence-based Ergonomics (EBE) approach, the collective sense of reality stems from a variety of systemic models, field data, narrative, and pictures. A variety of scholarly effort has been made to link basic insights drawn from the study of complexity raised by initiatives to define and implement ecological and sustainability approach for ergonomics. Sustainability can not only be described from a variety of perspectives, but new perspectives and evidences emerge into a view as one considers these models and systems at different scales within a nestled hierarchy.

Every year, researchers and scientists publish more than three million new articles in scientific journals, each adding height to an existing mountain of HFE evidence. This paper proposes a coherent review on a set of principles and assumptions with implications for structuring an ergonomics practice and design of solutions, for organizing its delivery, for carrying out the work environment and product design process, decision-making processes and for creating support tools for improved learning and teaching methodologies.

These underlying assumptions lead to an Evidence-Based Ergonomics approach to subsidize an HFE professional role that supports learning of specific domain knowledge and skills, and allow learning of self-instruction skills by explicitly integrating HFE research evidence with HFE reasoning and professional experience.

\section{Methodology}

This paper proposes a review on a set of principles and assumptions with implications for structuring ergonomics and human factors programs, for organizing its field application and research activities, for carrying out the work environment and product design process, and for creating support tools for improved interventions. These underlying assumptions lead to an Evidence-Based Ergonomics approach that: supports specific domain knowledge and skills, and by explicitly integrating HFE research evidence with HFE reasoning and professional experience to tackle issues in the workplace environment.

Ergonomics studies and their resulting interventions are part of the multidisciplinary process of systematically finding, appraising, and using contemporaneous research findings as the basis for practical decisions in the work environment. Although the formal assessment of ergonomic conditions and expert decision making using controlled trials in ergonomic studies have been advocated, the validation of these outcomes and concepts still need to be developed into a practical methodology. According to Meister (1999), the success in describing reality in human-technology relationship in ergonomic and human factors research is incomplete, no matter how detailed and comprehensive is the general statement to the overall problem (Silveira, 1999).

Increasingly practitioners and academy are looking to the strength and weight of scientific evidence on professional practice and cost effectiveness when defining resources allocation for their ergonomics solutions in the workplace. Several ergonomics and human factors studies 
outcomes have been considered in the literature based on their validity. Academic and professionals researchers are also using numerical data in explicitly-interpretative and contextual inquiry, such as naturalistic approach (Lincoln and Guba,1985). They advocate that validity of this information is required and, in this way, it may to encourage ergonomics professionals to adopt interventions that have been proven to be both practically and cost-effective, while disinvesting from practice that does not meet these objectives.

This paper focuses on the Concept Structure in Ergonomics as approached by Meister (1999) and the alternative way to develop knowledge structuring accompanied by supporting of thinking. Research and application are two different aspects of HFES, but these entities may be feed into the other in a loop arrangement when allied with human, technology and as an autonomous science.

\section{Evidence-based ergonomics model (EBE): proposing a reference model}

The proposition on this study is to combine the theoretical approach for Sustainability Science (Kumazawa et.al., 2009), the Taxonomy of the Human Factors and Ergonomics (HFE) discipline proposed by Meister (1999) as as shown in Table 2 and the framework for Evidence-Based Medicine (Evidence-Based Medicine Working Group, 1992, Woolf, 2001, Dawes \& Sampson, 2003), in an attempt to be applied in Human Factors and Ergonomics. Applications of ontologies are known in the field of medical research (for examples, see (Flanagan et al.,2005; Musen, 1992)) for lexicon or taxonomy-like descriptions of diseases or the genome, and computer science (for examples, see Antoniou \& Van Harmelen (2004)) for information retrieval and search methodologies.
By addressing the key challenges associated with knowledge structuring in HFES, it was scrutinized the key requirements as we assimilated Meister (1999) and Kumazawa et.al., (2009) proposals. A set of requirements was summarized (see Table 1) for the structuring of knowledge and to design a reference model. Based on this reference model we foresee the need for an ontology-based mapping tool as a solution to one level of the reference model. The Taxonomy of the HFE considers a basic category which includes HFE purpose, scope, functions, organization, research methods and disciplinary concepts.

First, it was identified the important requirements for HFES Concept structuring, as regarded by Meister (1999). Second, we develop an evidence-based ergonomics framework as a reference model composed of six levels based on these requirements. Third, we also devise an mapping tool using linguistic resources to translate human work, systems environment and the complexities inherent to their hierarchical relationships (Morin, 1996) to support future development at Level 2 of the reference model and for meeting the two major challenges for HFES, namely, identifying what problems should be addressed in HFE as an Autonomous Science itself, and proposing solutions by integrating concepts and methods applied in HFES for those problems. 
Table 1.

Requirements for EBE Model Construction

\begin{tabular}{|l|l|l|}
\hline $\begin{array}{c}\text { Sustainability Model } \\
\text { Requirements } \\
\text { (Kumazawa } \\
\text { et.al.,2009) }\end{array}$ & \multicolumn{1}{|c|}{$\begin{array}{c}\text { HFES Model } \\
\text { Requirements } \\
\text { (Meister,1999) }\end{array}$} & $\begin{array}{c}\text { Reasoning for Adoption in } \\
\text { Ontology HFES Model }\end{array}$ \\
\hline Versatility & Multidisciplinary & $\begin{array}{l}\text { Approach different context and } \\
\text { conditions and complexities. The } \\
\text { infrastructure consists of } \\
\text { elements, constructs, assumptions, } \\
\text { parameters, variables and } \\
\text { hypothesis }\end{array}$ \\
\hline Reusability & Validity & $\begin{array}{l}\text { Should be applied to as many } \\
\text { domains as possible without } \\
\text { restriction to a specific domain or } \\
\text { discipline }\end{array}$ \\
\hline Adjustability & Flexibility & $\begin{array}{l}\text { HFE problems are dynamic and } \\
\text { there are also a large number of } \\
\text { variables whose interactions } \\
\text { creates the phenomena which } \\
\text { HFE is concerned }\end{array}$ \\
\hline Availability & Research and \\
& Application Use & $\begin{array}{l}\text { The two primary HFE function is } \\
\text { research and application. } \\
\text { According to Meister(1999), HFE } \\
\text { considered as organizing } \\
\text { mechanism and its data should } \\
\text { fulfills the needs for researchers, } \\
\text { practitioners and users }\end{array}$ \\
\hline & &
\end{tabular}

\subsection{Use of the term model}

It is important not to restrict the sense of the term model as it has been used on this study. A system, environment, or expert performance can exist in reality or in the thinking of one or more individuals. Whatever elements of experience a practitioner has applied his/her past HFE experience through repetition or personal construction, together with whatever terminology, concepts or relationships he/she has observed or constructed between those elements, constitute the models that are available for use by that individual.

\subsection{Interaction with models}

For the remainder of the paper it will be adopted the term model in the three senses defined above, but it will drop reference to real objects as a separate term. When it is used the term model, it will automatically refer to both real things and representations of real things. EBE Model does not distinguish between these sources of information as long as they fill the specific field application need for which they are being employed. They are simply different sensible representations of the same set of systematic relations having differing amounts of information to supply the HFE professional. 
It is proposed an EBE model that consists of levels corresponding to these five kinds of information: HFE raw data, underlying static information structure, dynamic information reflecting individual perspectives, dynamic information organizing perspectives within context, and methodological information.

The EBE model is not a solution for structuring HFE knowledge; rather, it is a model that can be referred to when discussing knowledge structuring in human factors and in ergonomics application. It contributes to evaluating and understanding the differences and commonalities of knowledge structuring tools and methods from different disciplines to be proposed in the future by providing a common framework in which they are compared.

As shown in Fig. 1, the reference model consists of six levels. The bottom level, Level 1, is the HFE data level and stores raw data corresponding to the real world. Level 2, the ontology level, stores the ontology for evidences explaining and understanding the raw data at Level 1 . The ontology describes the concepts and relationships related to HFE that exist in the real world. Another function of the ontology is to provide a common vocabulary for promoting mutual understanding across HFE domains. Typical tasks performed at Level 1 consider metadata generation regarding virtual organization of the HFE raw data and efficient retrieval of the raw data using the metadata.

Kumazawa et al. (2009) adopted a classification to human thinking as proposed by Guilford (1950, 1967)) which classify it into divergent thinking and convergent thinking. It was assimilated these concepts into the present EBE reference model: divergent thinking is supported at Level 3 and convergent thinking is supported at Level 4.
Level 3 deals with dynamic information by reflecting individual perspectives. The main task supported by this level is the divergent exploration of the conceptual world realized at Level 2, which systematizes the concepts appearing in the HFE world. Divergent exploration in a large number of concepts uses divergent thinking across HFE related disciplines to guide researchers searching for interesting concepts/relationships that have been hidden in the conventional unstructured world. The ontology at Level 2 must contribute to such exploration. Divergent exploration can be performed by obtaining through conceptual chains, which are characterized by a multi-perspective, in the course of the selection of arbitrary concepts according to the researcher's intention. Many ways of tracing the conceptual chains may be needed for handling the various aspects of HFE.

After collecting such conceptual chains, the researcher would move on to a convergent thinking stage at Level 4 . The task of this level is 'context-based convergent thinking. At this level, the researcher can set a specific context of a problem that he or she actually treats and obtain 'multiple convergent conceptual chains' (Klein,2004) in accordance to the given context. Examples of contexts include the social and environmental settings of a specific problem, implemented or planned countermeasures and policies for solving a problem, and even trade-offs between different goals, such as health promotion and nuclear power production.

Level 5 consolidates the understanding of context-based convergent thinking and provide means to the researcher or practitioner to choose among multiple convergent conceptual chains in accordance to the given context and based on various viewpoints that help users to understand the HFE knowledge systematically across domains. 
At Level 6, using all of the information and knowledge at the sub levels, the researcher or practitioner will pursue essential problem-solving tasks, such as setting the conditions for solving a problem or searching for a new problem, as well as information integration, innovation, and the abduction of new hypotheses.
Level 6

Level 5

Level 4
HFE Problem Setting and Solution
The HFES Concept Structure

Context Based Convergence
Level 3
Divergent Exploration (multiperspective) Conceptual Structure

Ontology-Based HFES data Retrieval

\section{Level 1}

\section{HFES Raw Data}

Figure 1. Structure for Evidence-Based Ergonomics Model

\section{Evidence-based HFES data retrieval}

In integrated modeling ergonomics research, scientists from various disciplines can define a common conceptual schema that their models share. Wisner (1981) highlighted the importance of cultural diversity and different way to approach HFE issues. Wisner (1983) also has stated that "industrialization would thus more or less require a new kind of mental model." In this view, formal education and technical training, experience as well as culture are important factors in determining the formation of an adequate cognitive model. A way to address the complexity associated with integrated ergonomics assessment is to structure the knowledge by means of ontologies and knowledge bases.

By developing a common HFES ontology, i.e. ontology which may be shared by all related models to-be-integrated, serves as a knowledge-level specification of the joint conceptualization of the participating models and each model must adhere to the semantics of the concepts in the common ontology. It includes restrictions on the concepts and relationships between the concepts, but the internal specification of the 
knowledge in the model does not have to adhere to the common ontology (Gruber, 1993). This ontology captures HFES scientists' disciplinary knowledge in a declarative fashion, that can be ultimately translated in a practitioner and expert understandable format and be available for subsequent field application and research.

Applications of ontologies are known in the field of medical research (for examples, see (Flanagan et al., 2005; Musen, 1992) for lexicon or taxonomy-like descriptions of diseases or the genome, and computer science (for examples, see Antoniou \& Van Harmelen (2004)) for information retrieval and search methodologies.
The development of a common ontology in ergonomics by an interdisciplinary group of researchers is a complex, challenging and time-consuming task. Several authors such as Gruber, 1993; Holsapple \& Joshi, 2002; Musen, 1992) when addressing ontology have assigned that still remains a scientific challenge. Tools are available that help in ontology development (Farquhar, Fikes, Pratt, \& Rice, 1995) and to store the ontology once it was developed (Knublauch, 2005). To achieve ontological commitment, i.e. the agreement by multiple parties to adhere to a common ontology, when these parties do not have the same experiences and theories (Holsapple \& Joshi, 2002) a collaborative approach is suggested to be used.

Table 2.Meister's Proposed Taxonomy and example of EBE Model focus

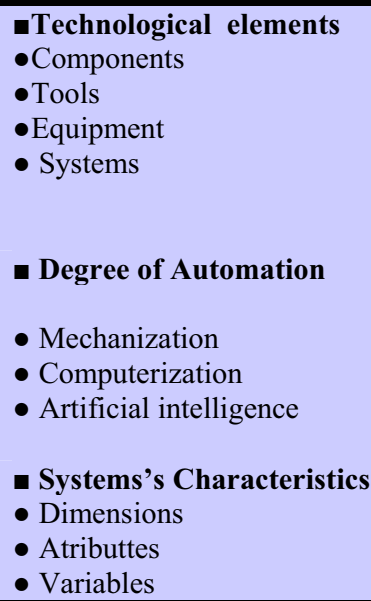

When developing the EBE Model it was identified the need to consider in the level 2 those requirements as shown in Table 1, such as versatility, interpretability and availability. It was observed that is required to choose generalized concepts that are not dependent on a specific scientific domain or field. In this sense, versatility can be achieved.
- Effects of technology on humans

- Changes in the role of human elements

- Changes in human behavior

- Technology-Organization Relationships

- Organizational strategies -Organizational variables
Future development of a mapping tool to support a database related HFE ontology is necessary. Availability can be achieved by preparing an exclusive website for the HFE ontology. Interpretability will be fulfilled to the extent that the HFE ontology and the mapping tool can help divergent thinking by explicating the knowledge structure. Using the ontology makes it easier to have a handle on the differences as well as the 
commonalities between disciplines and domains. For example, by comparing the maps generated from various viewpoints, a user could better understand the difference between his or her implicit assumptions and those of others. However, because interpretation depends on the particular mindset of each individual user, the ability of this function to achieve interpretability is limited.

\section{Future work and outlook}

The development of Evidence-Based Ergonomics Model is not yet complete. The current proposal of the ontology needs a cooperative work to congregate not only term's specifications and its basic definition, but also including variant definitions it may have.

The study concludes that the mapping tool may be useful enough to facilitate practitioners, researchers and users in general to get a more comprehensive picture of and their potential solutions; and providing an effective opportunity to come up with new resources and venues that might not be thought of without such a tool.

In closing it would be emphasized the key role of HFE education in this process. HFE as a science must nurture a generation of professionals and academics who are capable to get a step further not for establishing an unifying process, but to advocated HFE as transdisciplinary and multidisciplinary science available to everyone.

By supporting the path of multidisciplinary approach in implementing common and interchangeable concepts across domains and fostering the basis of this understanding. The expected design and capabilities of the devised tool is to store and retrieve information regarding HFES, to provide taxonomy of the HFE discipline.

This paper is therefore interested in supporting the development of HFE through systematically reviewing, appraising and applying field studies and research findings and the implementation ergonomics solutions that recognize the need for both global and local approaches to an integrated Human Factor and Ergonomics.

\section{References}

[1] Antoniou, G., \& van Harmelen, F. (2004). A Semantic Web Primer. Cambridge, Massachusetts; London, England: The MIT Press.

[2]Dawes \& Sampson, (2003) Knowledge management in clinical practice: a systematic review of information seeking behavior in physicians. Int $\mathbf{J}$ Med Inform. 2003 Aug;71(1):9-15

[3] Evidence-Based Medicine Working Group. Evidence based medicine. A new approach to teaching the practice of medicine. JAMA. 1992;268:2420-5.

[4] Flanagan, K., Stevens, R., Pocock, M., Lee, P., \& Wipat, A. (2005). Ontology for genome comparison and genomic rearrangements. Comparative and Functional Genomics, 5(6-7), 537-544.

[5] Goodman C. Literature searching and evidence interpretation for assessing health care practices. Stockholm: SBU, 1993.

[6] Gruber, T. R. (1993). A Translation Approach to Portable Ontology Specifications. Knowledge Acquisition, 5, 199-220.

[7] Guilford JP (1950) Creativity. Am Psychol 5:444454

[8] Guilford JP (1967). The nature of human intelligence. McGraw-Hill, New York

[9] Kaplan, R. Grabe, W. (2000). Applied linguistics and the annual review of applied linguistics. Annual Review of Applied Linguistics, 20 pp3-17, 2000.

[10] Klein JT (2004) Interdisciplinarity and complexity: an evolving relationship. Emerg Complex Organ 6(1-2):2-10; special double issue

[11] Knublauch, H. (2005). Protege OWL. Stanford: Stanford Medical Informatics.

[12] Kumazawa, T. ; Saito,O.,Kozaki. K. , Matsui, T. and Mizoguchi, R.(2009) Toward knowledge structuring of sustainability science based on ontology engineering. Sustain Sci (2009) 4:99-116 DOI $10.1007 / \mathrm{s} 11625-008-0063-\mathrm{z}$

[13] Meister. (1999). The History of Human Factors and Ergonomics, Lawrence Earlbaum Associates Publishers, Londo, UK.

[14] Montmollin, M. The future of ergonomis; hodge podge or new foundation? Le Travail Humain, tome 55, n. 2, p.171-81, 1992.

[15] Montmollin, M. L'ergonomie. Paris: La Découverte, 125 p., 1990.

[16] Moray, N. Ergonomics and the global problems of the twenty first century. Ergonomics, 38, 8, p.16911707,1995 
[17] Morin, E. (1996). Ciência com Consciência. Rio de Janeiro: Bertrand Brasil, 1996

[18] Musen, M. A. (1992). Dimensions of knowledge sharing and reuse. Computers and Biomedical Research, 25(5), 435-467.

[19] Silveira,D.M.C. (1999). Participation, Attitudes and the Design of Technical Systems, PhD. Thesis. University of Surrey.

[20] Uschold, M. and M. Gruninger. (1996). Ontologies: principles, methods and applications. The Knowledge Engineering Review, 11(2):93-136, 1996

[21]Woolf S. (2001) Evidence-based medicine: a historical and international overview. Proc R Coll Physicians Edinb. 2001;31 Suppl 9:39-41.

[22]Wisner, A . (1981) Vers une Anthropotechnologie. CNAM, Paris, $169 \mathrm{p}$.

[23] Wisner, A. (1983). Ergonomics or anthropology: A limited or wide approach to working condition in technology transfer. In Proceedings of the First International Conference On Ergonomics of Developing Countries, edited by Shahnavaz and Babri. Luleå, Sweden: Luleå Univ. of Technology. 